\title{
Baja calidad de interacción madre-hijo/a en lactantes en riesgo psicosocial se asocia con riesgo de retraso del desarrollo
}

\author{
Low quality of mother-child interaction in infants at psychosocial risk is associated \\ with risk of developmental delay
}

\author{
Victoria Binda $^{a}$, Francisca Figueroa-Leigh ${ }^{b}$, Marcia Olhaberry ${ }^{c}$
}

\author{
a Médico Familiar Mención Niño. Departamento de Medicina Familiar, Escuela de Medicina, Pontificia Universidad Católica de Chile \\ bMédico Familiar Mención Niño \\ cPsicóloga. Escuela de Psicología, Pontificia Universidad Católica de Chile
}

Recibido: 18 de junio de 2018; Aceptado: 29 de noviembre de 2018

\section{Resumen}

La infancia temprana es un período fundamental en el desarrollo de los niños y depende en gran parte de las interacciones que establecen con sus cuidadores principales. Objetivos: Evaluar la asociación entre riesgo de retraso del desarrollo psicomotor (RDSM) con calidad de interacción madre-hijo/a, síntomas depresivos postparto y otros factores relacionados al cuidado y contexto, en lactantes sanos en riesgo psicosocial. Pacientes y Método: Estudio transversal analítico en 181 madres en riesgo psicosocial y sus hijo/as menores de un año, atendidas en Atención Primaria de Salud. Se determinó la presencia de riesgo de RDSM utilizando Ages \& Stages Questionnaire y se estudió su asociación con calidad de interacción (CARE Index), síntomas depresivos postparto (Escala de Depresión Postparto Edimburgo) y otros factores relacionados al contexto y cuidado (cuestionario aplicado a la madre) mediante análisis bivariados y multivariados, ajustando por variables confundentes. Resultados: $20 \%$ de los lactantes presentó riesgo de RDSM. Se observó un mayor riesgo de presentar riesgo de RDSM, después de ajustar por variables de control predefinidas, con: baja calidad de la interacción madrehijo/a $(O R=2,46, p=0,03)$, lactancia materna exclusiva $(L M E)<6$ meses $(O R=2,58, p=0,01)$ y pareja no ayuda con el cuidado del niño $(O R=2,97, p=0,03)$. No se observó una asociación significativa con síntomas depresivos postparto. Conclusiones: En lactantes sanos en riesgo psicosocial se asocian a mayor riesgo de RDSM una baja calidad interacción madre-hijo/a, LME $<6$ meses y no involucramiento del padre en el cuidado del hijo/a.
Palabras clave:

Desarrollo psicomotor; interacción madrehijo/a; depresión postparto; factores de riesgo; lactancia materna; factores psicosociales

Correspondencia:

Victoria Binda

vbinda@uc.cl 


\begin{abstract}
Early childhood is a fundamental period in children's development and depends largely on their interactions with their main caregivers. Objectives: To evaluate the association between risk of psychomotor developmental delay (PDD) with mother-child interaction quality, postpartum depressive symptoms, and other factors related to care and environment in healthy infants at psychosocial risk. Patients and Method: Analytical cross-sectional study in 181 mothers at psychosocial risk and their children aged under one year seen in Primary Health Care. The presence of risk of PDD was determined using the Ages \& Stages Questionnaire and its association with interaction quality (CARE Index), postpartum depressive symptoms (Edinburgh Postpartum Depression Scale), and other factors related to environment and care (questionnaire applied to the mother) was studied through bivariate and multivariate analyses, adjusting for confusing variables. Results: $20 \%$ of infants were at risk of PDD. There was an increased risk of presenting risk of PDD after adjusting for predefined control variables with: low-quality mother-child interaction $(\mathrm{OR}=2.46, \mathrm{p}=0.03)$, exclusive breastfeeding $(\mathrm{EBF})<6$ months $(\mathrm{OR}=2.58, \mathrm{p}=0.01)$, and partner does not help with childcare $(\mathrm{OR}=2.97, \mathrm{p}=$ 0.03). No significant association was observed with postpartum depressive symptoms. Conclusions: In healthy infants at psychosocial risk, low-quality mother-child interaction, EBF $<6$ months, and the non-involvement of the father in the childcare are associated with a higher risk of PDD.
\end{abstract}

\section{Keywords:}

Psychomotor development; mother-child interaction; postpartum depression; risk factors; breastfeeding; psychosocial factors

\section{Introducción}

El desarrollo infantil es un proceso de maduración que depende de la interacción del niño/a con otras personas $^{1}$. El período prenatal hasta los $4-5$ años es clave para el desarrollo físico, cognitivo y socioemocional, ya que brinda las bases para el desarrollo a lo largo de la vida $^{2}$.

La prevalencia de retraso del desarrollo psicomotor (RDSM) en niños menores de 3 años en los países desarrollados es entre el 12 al 16\%\%3,. En Chile, según la última encuesta de Calidad de Vida y Salud 2016-17 la prevalencia es de un 9,9\%, en niños menores de 5 años, presentando una disminución significativa en los últimos años.

Es de gran relevancia detectar precozmente el RDSM, ya que aquellos niños que no reciben intervención temprana tienen mayor probabilidad de presentar fallo en el sistema escolar, problemas de conducta, baja autoestima, problemas de salud mental y social como delincuencia, desempleo y pobreza ${ }^{6}$, con un impacto social y económico para toda la sociedad ${ }^{7}$. A su vez la detección e intervención temprana han demostrado efectividad en la mejoría de distintos resultados como: rendimiento escolar, calidad de los empleos, conducta criminal y deserción escolar, entre otros, además de una probada disminución de los costos asociados 8 .

Se han descrito diferentes factores de riesgo para presentar RDSM ${ }^{9}$; algunos dependen de las características del niño/a como: sexo masculino, nacimiento prematuro, bajo peso al nacer y presencia de enfermedades crónicas; otros, de las características de sus cuidadores principales como: pobre calidad de la interacción con el niño/a, dificultades en la relación en- tre los padres, problemas de salud mental, bajo nivel educacional y padres adolescentes. Y por último existen factores de riesgo dependientes del contexto como: pobreza, escaso apoyo a la paternidad, baja calidad del cuidado extra-hogar y ambientes poco estimulantes para el desarrollo infantil. Un estudio chileno en preescolares identificó que en aquellas familias donde hay mayor pobreza, no hay asistencia a educación preescolar y/o las madres son analfabetas, hay mayor riesgo de $\mathrm{RDSM}^{10}$. Otro estudio en preescolares chilenos muestra mayor RDSM en niños atendidos en la red de salud pública comparado con en el sistema privado ${ }^{2}$. Un estudio de cohorte realizado en Estados Unidos ${ }^{11}$ demostró que uno de los predictores más relevantes para un adecuado DSM era la calidad de la interacción madre-hijo/a: esto es, entre más sensible, responsiva, atenta y cognitivamente estimulante se mostraba la madre durante las interacciones, mejor eran los resultados obtenidos en las evaluaciones del DSM de los niños. Es más, una baja calidad de la interacción se ha postulado como el posible mecanismo por el cual las características de los cuidadores principales y el contexto impactan finalmente en el desarrollo del niño/ $\mathrm{a}^{12}$.

Un ejemplo de esto es la depresión postparto (DPP), considerada como uno de los factores más influyentes en la calidad de la interacción madre-hijo/a y en el desarrollo infantil ${ }^{13}$. Ésta afecta la calidad de la interacción y posiblemente impacta el DSM a través de este mecanismo. La DPP es una patología prevalente, reportada entre el 20 y $37 \%{ }^{14,15}$ de la población chilena, llegando hasta un $50 \%$ en sectores de bajo nivel socioeconómico ${ }^{16}$, por lo que resulta relevante su consideración para la evaluación del RDSM.

Considerando los antecedentes anteriormente ex- 
puestos, conocer los factores asociados a RDSM infantil es de suma importancia para la pesquisa temprana de niños en riesgo y para intervenir sobre aquellos factores modificables, que permitan evitar el impacto del RDSM a largo plazo.

\section{Objetivos}

Evaluar la asociación entre riesgo de RDSM y a) calidad de la interacción madre-hijo/a; b) síntomas depresivos post parto y c) otros factores relacionados al cuidado y contexto, en lactantes sanos en riesgo psicosocial atendidos en Atención Primaria de Salud (APS) en Santiago de Chile.

Se espera que tanto la baja calidad de la interacción como la presencia de síntomas depresivos postparto se asocien de manera significativa con RDSM, así como identificar otros factores de riesgo relacionados al cuidado y contexto del niño/a.

\section{Pacientes y Método}

\section{Diseño del estudio}

Estudio transversal analítico. Los datos utilizados para el presente estudio fueron obtenidos de la muestra basal de un estudio clínico randomizado (FONIS SA12 | 2089), cuyo objetivo fue evaluar la efectividad de una intervención grupal ${ }^{17}$ para aumentar la sensibilidad materna. Este estudio se encuentra en fase de análisis de resultados.

\section{Población de referencia}

Se obtuvo una muestra de 181 madres en riesgo psicosocial y sus hijo/as menores de un año, atendidas en los centros de Salud Familiar (CESFAM) Juan Pablo II y El Roble, de la comuna de La Pintana, Santiago, Chile, durante los años 2013-2015. Considerando una prevalencia poblacional del $17 \%$ de riesgo de RDSM, cifra reportada en el estudio de validez de Ages \& Stages Questionnaire (ASQ) en Chile a los 8 meses de edad $^{18}$, esta muestra permite estudiar la prevalencia de riesgo de RDSM y sus asociaciones, con una precisión del 5,41\% y un nivel de confianza del 95\%.

Los criterios de inclusión fueron: Madres en riesgo psicosocial con hijos/as entre 2 y 12 meses de edad. La definición de riesgo psicosocial es la utilizada en APS en Chile , definido como la presencia de uno o más factores de riesgo psicosocial detectados en el primer control prenatal mediante la a escala de Evaluación Psicosocial Abreviada (EPsA) ${ }^{19}$, los factores de riesgo considerados son: primer control posterior a las 20 semanas, $<6$ años de estudio escolar, edad $<18$ años, consumo de sustancias durante el embarazo (tabaco , alcohol o drogas), víctima de violencia, conflictos con la maternidad, insuficiente apoyo social o familiar y síntomas depresivos. Los criterios de exclusión fueron: madres con patología de salud mental severa (esquizofrenia, retardo mental y trastorno de ánimo con ideación suicida activa), niños con patologías biomédicas importantes (genopatías, cardiopatías severas y prematurez extrema) y/o problemas psicosociales severos (antecedentes de institucionalización de otros hijos/as, denuncias por abuso sexual y/o maltrato infantil).

\section{Procedimiento}

Se contactó telefónicamente a las madres que cumplían con los criterios de inclusión para invitarlas a participar. El reclutamiento se realizó en forma sucesiva de manera de completar el número de participantes. La entrevista de ingreso fue realizada en el CESFAM respectivo por profesionales trabajadores del mismo, que colaboraron con el estudio. Todas las madres que acudieron a la entrevista de ingreso accedieron a participar y firmaron el consentimiento informado, luego completaron los instrumentos de evaluación.

\section{Instrumentos}

Para la evaluación de riesgo del DSM se utilizó Ages \& Stages Questionnaire (ASQ Segunda edición, traducido al español $)^{20}$. Si uno o más áreas evaluadas resulta $<2$ DS el tamizaje es positivo y se considera como riesgo de RDSM. En Chile esta escala se encuentra validada con buenas propiedades psicométricas ${ }^{18}$.

Para la evaluación de la calidad de la interacción diádica se utilizó CARE-Index Infants ${ }^{21}$, que consta de la observación y microanálisis de una videograbación de 3 min de juego libre de la díada. Entrega un puntaje de sensibilidad materna en una escala de 0 a 14 (mejor sensibilidad a mayor puntaje). Puntajes de 0 a 6 se consideran con baja calidad de la interacción. Los videos fueron evaluados por tres codificadoras que fueron entrenadas por la autora del instrumento y obtuvieron el test de confiabilidad. La correlación intraclase entre las codificadoras fue $>0,9, \mathrm{p}<0,001$. La codificación se realizó en forma independiente y fue ciega al resto de las mediciones.

Para la evaluación de síntomas depresivos postnatales se utilizó la Escala de Depresión Postnatal de Edimburgo $^{22}$, consta de 10 preguntas con 30 puntos como puntaje máximo. Está validada en Chile ${ }^{16}$ con buenas propiedades psicométricas. Se utilizó el punto de corte de $\geq 10$ puntos para considerar la presencia de síntomas depresivos postparto.

Para la evaluación de otros factores de riesgo de RDSM las participantes completaron un cuestionario de datos sociodemográficos y cuidado, incorporando características del niño, de los cuidadores y del contexto que pudieran estar asociados a la presencia de RDSM (tabla 1). 
Tabla 1. Características sociodemográficas y de cuidado de la muestra y su asociación con riesgo de retraso del desarrollo psicomotor en análisis bivariado

\begin{tabular}{|c|c|c|c|c|}
\hline \multirow[t]{2}{*}{ Característica } & \multirow{2}{*}{$\frac{\text { Total }(n=181)}{n(\%)}$} & \multicolumn{3}{|c|}{ Asociación con riesgo de RDSM } \\
\hline & & $\mathrm{p}$ & OR & IC 95\% \\
\hline \multicolumn{5}{|l|}{ Del niño/a } \\
\hline Edad $\leq 6 \mathrm{~m}$ & $66(36,5)$ & 0,02 & 2,28 & $1,08-4,7$ \\
\hline Sexo masculino & $92(50,8)$ & $0,79 \mathrm{NS}$ & 1,1 & $0,5-2,2$ \\
\hline Hijo Único & $77(42,7)$ & $0,56 \mathrm{NS}$ & 1,2 & $0,5-2,5$ \\
\hline Edad Gestacional $\leq 37$ semanas & $34(18,6)$ & 0,34 NS & 1,5 & $0,6-3,7$ \\
\hline Pequeño para edad gestacional & $13(7,1)$ & 0,63 NS & 0,68 & $0,14-3,2$ \\
\hline Hospitalización $\mathrm{RN} \geq 4$ días & $42(23,2)$ & $0,11 \mathrm{NS}$ & 1,8 & $0,8-4,1$ \\
\hline Hospitalizaciones posteriores al nacimiento & $35(19,3)$ & 0,98 NS & 0,9 & $0,39-2,49$ \\
\hline LME $<6$ meses o edad al ingreso al estudio & $59(32,6)$ & 0,015 & 2,48 & $1,17-5,25$ \\
\hline Nunca recibió LM & $23(12,7)$ & 0,06 NS & 1,15 & $0,95-1,3$ \\
\hline \multicolumn{5}{|l|}{ De los cuidadores } \\
\hline Embarazo adolescente & $34(18,8)$ & 0,2 NS & 0,49 & $0,16-1,5$ \\
\hline Embarazo no planificado & $128(70,7)$ & $0,15 \mathrm{NS}$ & 1,6 & $0,7-3,2$ \\
\hline Padre no vive en el hogar & $81(44,8)$ & $0,27 \mathrm{NS}$ & 1,2 & $0,32-1,28$ \\
\hline Padre no ayuda con el cuidado del niño & $130(71,8)$ & 0,03 & 2,94 & $1,07-8,05$ \\
\hline Educación escolar incompleta madre & $102(56,4)$ & $0,38 \mathrm{NS}$ & 0,72 & $0,34-1,5$ \\
\hline Antecedente depresión previa madre & $82(45,3)$ & $0,57 \mathrm{NS}$ & 1,23 & $0,5-2,5$ \\
\hline \multicolumn{5}{|l|}{ Del contexto } \\
\hline Hacinamiento & $60(33,1)$ & $0,73 \mathrm{NS}$ & 0,87 & $0,39-1,9$ \\
\hline Violencia dentro del hogar & $11(6,1)$ & $0,16 \mathrm{NS}$ & 2,4 & $0,67-8,8$ \\
\hline Consumo drogas o alcohol significativo en el hogar & $22(12,2)$ & 0,14 NS & 2,04 & $0,7-5,4$ \\
\hline Nadie ayuda con cuidado del niño & $45(24,9)$ & 0,09 NS & 1,9 & $0,89-4,3$ \\
\hline Asistencia a sala cuna & $11(6,1)$ & $0,08 \mathrm{NS}$ & 0,9 & $0,88-0,96$ \\
\hline
\end{tabular}

LME: lactancia materna exclusiva; RDSM (retraso del desarrollo psicomotor), OR (Odds ratio), IC 95\% (Intervalo de confianza al 95\%), NS (no significativo $p>0,05$ ).

\section{Consideraciones éticas}

El estudio contó con la aprobación y cumplió con las exigencias de los Comités de Ética de la Pontificia Universidad Católica de Chile y el Servicio de Salud Metropolitano Sur Oriente.

\section{Análisis estadístico}

La descripción de la muestra se realizó mediante medias, desviación estándar y proporciones. Se usaron pruebas de chi cuadrado, razón de disparidad (OR) y pruebas $t$ para muestras independientes para los análisis bivariados, considerando significativo un valor $\mathrm{p}<0,05$. Se realizó un análisis de regresión logística multivariado con aquellas variables de asociación significativa para medir la magnitud del efecto, ajustando por las siguientes variables de control conocidas como factores de riesgo para RDSM, definidas como : sexo masculino, madre adolescente $(<18$ años al ingreso del embarazo), educación escolar materna incompleta $(<12$ años de estudio), prematurez (edad gestacional $\leq 37$ semanas), hospitalización del recién nacido ( $R N$ ) $\geq 4$ días y edad del niño $\leq 6$ meses. El análisis estadístico se realizó con el software SPSS versión 17.

\section{Resultados}

La media de edad de las madres fue 25,3 años, (rango de 14 a 46 años, $\mathrm{DE}=7,07$ ), la media de edad de los niños fue 7.39 meses (rango de 2 a 12 meses, $\mathrm{DE}=2,12$ ). Las características sociodemográficas y de cuidado de la muestra se presentan en la tabla 1.

Un 20,1\% $(n=36)$ de los niños presentaron riesgo de RDSM, un $57,3 \%(\mathrm{n}=102)$ de las díadas presentó baja calidad de la interacción y el $46,7 \%$ de las madres $(\mathrm{n}=84)$ presentó síntomas depresivos postparto.

Un 25,7\% de las díadas con baja calidad de la interacción, presentó riesgo del RDSM comparado con un $13,4 \%$ de aquellas con calidad de interacción adecuada (Figura 1), esta diferencia resulta estadísticamente significativa $\left(\chi^{2}=4,07, \mathrm{p}=0,04, \mathrm{OR}=2,25\right.$, IC $95 \%$ [1,01-5,02].

Un 25\% de los hijos de madres con síntomas depresivos presentó riesgo de RDSM, comparado con un $15,8 \%$ de los hijos de madres sin síntomas depresivos, esta diferencia no es estadísticamente significativa $\left(\chi^{2}=2,35 \mathrm{p}=0,12, \mathrm{OR}=2,25\right.$, IC $95 \%[0,84-3,72]$.

Como se observa en la tabla 1 , de las variables es- 
Tabla 2. Análisis multivariado de los factores asociados a riesgo de retraso del desarrollo psicomotor en lactantes en riesgo psicosocial

\begin{tabular}{lccc}
\hline Factores de riesgo & OR a & IC 95\% & p \\
\hline Baja calidad de interacción madre-hijo/a & 2,46 & $1,06-5,71$ & 0,03 \\
LME < 6 meses o edad al ingreso al estudio & 2,58 & $1,17-5,66$ & 0,01 \\
Padre no ayuda con el cuidado del niño & 2,97 & $1,06-8,29$ & 0,03 \\
\hline
\end{tabular}

LME: lactancia materna exclusiva. ORa (Odds ratio ajustado), IC 95\% (Intervalo de confianza al 95\%) y valor p obtenidos con modelos de regresión logística, ajustando por: sexo masculino, madre adolescente, educación escolar materna incompleta, prematurez, hospitalización del RN y edad $\leq 6$ meses

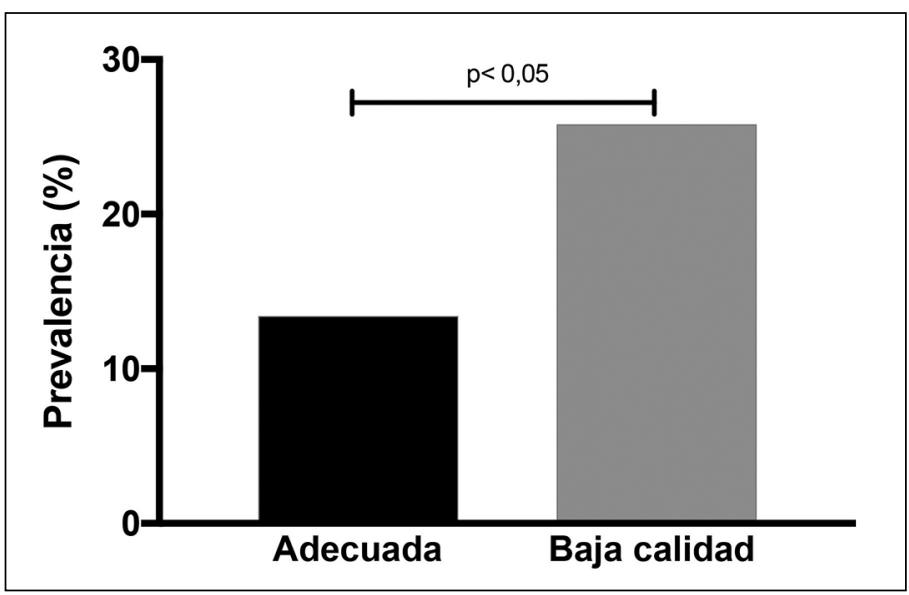

Figura 1. Prevalencia de riesgo de retraso del desarrollo psicomotor según calidad de interacción madre-hijo/a $(n=181)$.

tudiadas mediante el cuestionario sociodemográfico y de cuidado, en el análisis bivariado se asociaron significativamente con riesgo de RDSM: lactancia materna exclusiva (LME) $<6$ meses o edad de ingreso al estudio, pareja no ayuda con el cuidado del niño y edad del niño $\leq 6$ meses.

Al realizar el análisis multivariado por medio de regresión logística, ajustando por posibles variables confundentes predefinidas: sexo masculino, madre adolescente, educación escolar materna incompleta, prematurez, hospitalización del RN y edad $\leq 6$ meses, se obtuvo que incrementaron el riesgo de presentar riesgo de RDSM una baja calidad de la interacción madre-hijo/a, LME $<6$ meses y pareja no ayuda con el cuidado del niño (tabla 2).

\section{Discusión}

Este estudio muestra una asociación positiva entre baja calidad de la interacción madre-hijo/a con la presencia de riesgo de RDSM en lactantes sanos en riesgo psicosocial. La baja calidad de la interacción madre- hijo/a aumenta en 2,4 veces las chances de presentar riesgo de RDSM ajustando por variables conocidas por su asociación con RDSM. No se observó una asociación significativa entre síntomas depresivos postparto y riesgo de RDSM. Otros factores de riesgo detectados en este estudio fueron: $\mathrm{LME}<6$ meses o edad de ingreso al estudio y falta de involucramiento del padre en el cuidado del hijo/a.

Se comprueba en díadas chilenas que la presencia de baja calidad de la interacción vincular madre-hijo/a el primer año de vida, se asocia a mayor presencia de problemas en el desarrollo infantil en lactantes. Esta asociación es concordante con la literatura internacional: la parentalidad poco sensible el primer año de vida se asocia con peores resultados cognitivos y sociales hasta al menos los 48 meses de edad ${ }^{23,24}$, por otro lado, la calidad de la interacción presenta alta estabilidad desde el primer año de vida ${ }^{12,25}$, influyendo desde tan temprana edad en los resultados de desarrollo a futuro.

La importancia del padre en el desarrollo infantil cada vez se encuentra más estudiada ${ }^{26}$, se reconocen los hogares monoparentales como factor de riesgo para $\mathrm{RDSM}^{11}$. En nuestro estudio no fue la presencia del padre en el hogar la variable asociada a RDSM, sino el involucramiento del padre con el cuidado del niño, esto podría asemejarse a los estudios que evalúan una buena calidad de la interacción padre-hijo/a ${ }^{23}$ como muy relevante para los resultados en desarrollo.

La ausencia de asociación entre síntomas depresivos post parto y RDSM en nuestra muestra puede estar dada principalmente por dos causas: 1) falta de n suficiente para encontrar la diferencia; 2) al ser un estudio transversal y en lactantes pequeños, la presencia de síntomas depresivos en la madre aun no alcanza a afectar visiblemente el DSM del niño, pero su efecto podría encontrarse en los niños/as posteriormente, como se observó en el estudio de cohorte de Lung et al. ${ }^{27}$, que no mostró asociación entre salud mental materna y DSM a los 18 meses de edad, pero si a los 36 meses.

Es importante considerar que los factores de cuidado y contexto están interrelacionados ${ }^{11}$, una madre que cuenta con un padre que apoya en el cuidado del hijo, presenta menor estrés y menos posibilidades de presentar síntomas depresivos. De esta manera es más probable que tenga interacciones de buena calidad con su hijo/a, que resulten estimulantes para su desarrollo psicomotor.

En relación a los factores de cuidado evaluados resulta muy interesante la asociación encontrada entre riesgo de RDSM y LME $<6$ meses, lo cual también está avalada por la literatura. Existen estudios que demuestran una asociación lineal tipo "dosis respuesta" entre la duración de la LME y el desarrollo motor y cognitivo, siendo esta relación independiente de las características de los niños/as y sus padres ${ }^{28,29}$. 


\section{Limitaciones}

Para el presente estudio se utilizó la escala de ASQ en su segunda edición en español, esta no es la misma versión adaptada y validada de este cuestionario en Chile $^{20}$, ya que, al momento del reclutamiento del estudio, los resultados de la validación aún no se encontraban disponibles. No se cuenta con una evaluación directa de la interacción del padre u otro cuidador relevante para el niño/a, que sería lo ideal, ya que está estudiada su relevancia en el desarrollo y que puede actuar como buffer en caso de que la interacción con la madre no sea adecuada ${ }^{23}$. Existen limitaciones dadas por ser un estudio transversal: solo observamos asociación entre las variables, no sabemos cómo influye la calidad de la interacción y síntomas depresivos observados en el DSM de estos niños a futuro, aunque la literatura avala su estabilidad en el tiempo. Tampoco sabemos si la baja calidad de la interacción observada está en parte dada por la presencia de RDSM, en la literatura se postula que esta influencia es recíproca, ya que la interacción resulta más desafiante con un niño que no presenta el desarrollo esperado ${ }^{25}$. Los resultados provienen de una muestra de bajo nivel socioeconómico y en riesgo psicosocial, por lo que no son generalizables a toda la población.

Los resultados de este estudio resultan relevantes para las políticas públicas de nuestro país, siendo una muestra proveniente de un contexto de riesgo psicosocial es necesario conocer cuáles son los factores asociados a RDSM, sobre todo aquellos que son modificables por medio de intervenciones adecuadas, como son los encontrados en este estudio: promoción de la calidad de la interacción, incentivar el involucramiento del padre en el cuidado del niño y promover cuando sea posible la LME por 6 meses. Por otra parte, es necesario incorporar dentro de las atenciones realizadas a los niños detectados con rezago o RDSM la evaluación de la calidad de la interacción madre-hijo/a y ofrecer intervenciones que promuevan la sensibilidad materna, para lograr la mejoría en los resultados del desarrollo. Existen diversos estudios que muestran que las intervenciones pueden ser efectivas en aumentar la sensibilidad materna ${ }^{30}$.

Se requieren estudios que comprueben que inter- venciones enfocadas en el aumento de la sensibilidad materna dirigidas a niños con RDSM muestran mejores resultados en su desarrollo a largo plazo.

\section{Responsabilidades Éticas}

Protección de personas y animales: Los autores declaran que los procedimientos seguidos se conformaron a las normas éticas del comité de experimentación humana responsable y de acuerdo con la Asociación Médica Mundial y la Declaración de Helsinki.

Confidencialidad de los datos: Los autores declaran que han seguido los protocolos de su centro de trabajo sobre la publicación de datos de pacientes.

Derecho a la privacidad y consentimiento informado: Los autores han obtenido el consentimiento informado de los pacientes y/o sujetos referidos en el artículo. Este documento obra en poder del autor de correspondencia.

\section{Conflicto de interés}

Los autores no declaran conflicto de interés.

\section{Agradecimientos}

Agradecemos a las madres y niños/as que participaron en este estudio, a los CESFAM Juan Pablo II y El Roble de la comuna de La Pintana y a Patricia López, Paula Ramírez y María Isabel Moya que colaboraron con la investigación.

\section{Financiamiento}

El presente trabajo forma parte del proyecto FONIS SA12 2089. FONIS no influyó en el diseño del estudio; en la recolección, análisis o interpretación de los datos; ni en la preparación, revisión o aprobación del presente manuscrito. 


\section{Referencias}

1. Black MM, Walker SP, Fernald LC, et al. Early childhood development coming of age: science through the life course. For the Lancet Early Childhood Development Series Steering Committee. Lancet 2017;389:77-90.

2. Bedregal P, Hernández V, Mingo MV, et al. Desigualdades en desarrollo infantil temprano entre prestadores públicos y privados de salud y factores asociados en la Región Metropolitana de Chile. Rev Chil Pediatr. 2016;87(5):351-8.

3. Guevara J, Gerdes M, Localio R, et al. Effectiveness of developmental screening in an Urban Setting. Pediatrics. 2013;131(1):30-7.

4. Vitrikas K, Savard D, Bucaj M. Developmental Delay: When and How to Screen. Am Fam Physician. 2017;96(1):36-43.

5. Encuesta nacional de salud 20162017. Segunda entrega de resultados. Departamento de Epidemiología, Ministerio de Salud Chile. Enero 2018. Última visita: 14/06/2018. Disponible en: http://www.minsal.cl/wp-content/ uploads/2018/01/2-Resultados ENS_ MINSAL_31_01_2018.pdf

6. Bedregal P, Scharager J, Breinbauer C, Solari J, Molina H. El desarrollo de un modelo para la evaluación de rezagos del desarrollo infantil de Chile. Rev Med Chil. 2007;135(3):403-5.

7. Tough SC, Siever JE, Leew S, Johnston DW, Benzies K, Clark D. Maternal mental health predicts risk of developmental problems at 3 years of age: Follow up of a community-based trial. BMC Pregnancy Childbirth. 2008;8(1):16. doi:10.1186/1471-2393-8-16.

8. Anderson LM, Shinn C, Fullilove MT, et al. The effectiveness of early childhood development programs. A systematic review. Am J Prev Med. 2003;24(3): 32-46.

9. Parfitta Y, Pikea A, Ayersb S. Infant Developmental Outcomes: A Family Systems Perspective. Inf Child Dev. 2014; 23:353-73.

10. Schonhaut L, Rojas P, Kaempffer AM. Factores de riesgo asociados a déficit del desarrollo psicomotor en preescolares de nivel socioeconómico bajo: Comuna urbano rural, Región Metropolitana, 2003. Rev Chil Pediatr. 2005;76(6):58998.

11. NICHD Early Child Care Research Network. Child Care and Child Development: Results from the NICHD Study of Early Child Care and Youth Development. Guilford Press, New York, NY, USA. 2005.

12. Lugo-Gil J, Tamis-LeMonda CS. Family resources and parenting quality: links to children's cognitive development across the first 3 years. Child Dev 2008; 79(4):1065-85.

13. Stein A, Pearson RM, Goodman SH, et al. Effects of perinatal mental disorders on the fetus and child. Lancet. 2014; 384:1800-19.

14. Jadresic E. Depresión postparto. Rev Chil Neuro-Psiquiat. 1990;28:147-58.

15. Jadresic E, Jara C, Miranda M, Arrau $\mathrm{B}$, Araya, R. Trastornos emocionales en el embarazo y el puerperio: estudio prospectivo de 108 mujeres. Rev Chil Neuro-Psiquiat. 1992;30:99-106.

16. Jadresic E, Araya R. Prevalencia de depresión posparto y factores asociados en Santiago, Chile. Rev Med Chile 1995; 123: 694-9.

17. Figueroa-Leight F, Binda V, Santelices $P$. Enhancing early attachment: Design and pilot study of an intervention for primary health care dyads. J Child Health Care. 2013;17:82-91.

18. Armijo I, Schonhaut L, Cordero M. Validation of the Chilean version of the Ages and Stages questionnaire (ASQ-CL) in community health settings. Early Hum Dev. 2015;91:671-6.

19. Manual Apoyo y Seguimiento del Desarrollo Psicosocial de Niños y Niñas de 0 a 6 años. Primera Edición: septiembre 2008. MINSAL. Última visita: 14-06-2018. Disponible en: http:// www.crececontigo.gob.cl/wp-content/ uploads/2010/01/Manual-para-elApoyo-y-Seguimiento-del-DesarrolloPsicosocial-de-los-Ninos-y-Ninas-de-0-a6-Anos-2008.pdf.

20. Squires J, Bricker D, Twombly E, et al. Ages \& Stages Questionnaires ${ }^{\circledR}$, Second Edition (ASQ-2 ${ }^{\mathrm{TM}}$ ): A ParentCompleted Child Monitoring System.
Second Edition. Translated from English. Baltimore: Brookes Publishing Company, 1999.

21. Crittenden P. CARE-Index para Infantes (Nacimiento-15 meses). Manual de Codificación. 2006. Family Relations Institute, Miami, USA. (Manuscrito no publicado).

22. Cox JL, Holden JM, Sagovsky R. Detection of postnatal depression: development of the 10 item Edinburgh Postnatal Depression Scale. Br J Psychiatry. 1987;150:782-6.

23. Malmberg L-E, Lewis S, West A, Murray E, Sylva K, Stein A. The influence of mothers' and fathers' sensitivity in the first year of life on children's cognitive outcomes at 18 and 36 months. Child Care Health Dev. 2016;42: 1-7.

24. Laundry SH, Smith KE, Swank PR, Assel $\mathrm{MA}$, Vellet $\mathrm{S}$. Does early responsive parenting have special importance for children's development or is consistency across early childhood necessary? Dev Psychol. 2001;37:387-403.

25. Ciciolla L, Crnic KA, West SG. Determinants of Change in Maternal Sensitivity: Contributions of Context, Temperament, and Developmental Risk. Parent Sci Pract. 2013;13(3): 178-95.

26. Pancsofar N, Vernon-Feagans L. Fathers' early contributions to children's language development in families from low-income rural communities. Early Child Res Q. 2010;25:450-63.

27. Lung FW, Chiang TL, Lin SJ, Shu BC. Parental mental health and child development from six to thirty-six months in a birth cohort study in Taiwan. J Perinat Med. 2009;37(4):397-402.

28. Weisstaub G, Schonhaut L, Salazar G. Lactancia materna, desarrollo motor y obesidad, ¿Existe asociación causal? Rev Chil Pediatr. 2017;88(4):451-7.

29. Horta BL, Loret de Mola C, Victora CG. Breastfeeding and intelligence: a systematic review and meta-analysis. Acta Pædiatr. 2015;104(467):14-9.

30. Rayce SB, Rasmussen IS, Klest SK, Patras J, Pontoppidan M. Effects of parenting interventions for at-risk parents with infants: a systematic review and metaanalyses. BMJ Open. 2017;7(12):e015707. 